\title{
Hepatic Resection after Initial Transarterial Chemoembolization Versus Transarterial Chemoembolization Alone for the Treatment of Hepatocellular Carcinoma: A Meta-analysis of Observational Studies
}

\author{
Yu-Long Tang ${ }^{1,2 \& *}$, Xing-Shun $\mathrm{Qi}^{1,3 \& *}$, Xiao-Zhong Guo ${ }^{3 *}$
}

\begin{abstract}
Background: There is no consensus regarding the selection of treatment options for hepatocellular carcinoma (HCC) after initial transarterial chemoembolization (TACE). This meta-analysis aimed to explore the survival benefit of hepatic resection after initial TACE for the treatment of HCC. Materials and Methods: We searched three major databases to identify all relevant papers comparing the outcomes of hepatic resection after initial TACE versus TACE alone for the treatment of HCC. Hazard ratios (HRs) with 95\% confidence intervals (95\% CIs) were calculated to evaluate the survival benefit of hepatic resection after initial TACE over TACE alone. Results: Three of 2037 initially identified papers were included. All of them were cohort studies from Asia. There was a significantly better overall survival $(O S)$ in patients undergoing hepatic resection after initial TACE than in those undergoing TACE alone $(\mathrm{HR}=0.63,95 \% \mathrm{CI}=0.52-0.76, P<0.00001)$. The heterogeneity among studies was not statistically significant $\left(P=0.96 ; I^{2}=0 \%\right)$. Conclusions: Hepatic resection could improve the $O S$ of HCC patients treated with initial TACE. Further randomized controlled trials should be necessary to identify the target population for the sequential use of hepatic resection after initial TACE and to compare the outcomes between patients undergoing hepatic resection after initial TACE session versus those undergoing TACE alone.
\end{abstract}

Keywords: Hepatocellular carcinoma - transarterial chemoembolization - resection - surgery - survival

Asian Pac J Cancer Prev, 16 (17), 7871-7874

\section{Introduction}

Hepatocellular carcinoma (HCC) is one of the most prevalent and lethal cancer in the world (El-Serag, 2011; Forner et al., 2012). At present, the European Association for the Study of the Liver (EASL) and the American Association for the Study of Liver Disease (AASLD) guidelines recommend the use of the Barcelona Clinic Liver Cancer (BCLC) staging system as the reference therapeutic algorithm of HCC (Bruix and Sherman, 2011; 2012). Notably, most of HCC patients are diagnosed at the late stage, and lose the opportunity of curative therapy, such as liver transplantation, hepatic resection, and radiofrequency ablation (RFA). According to the EASL and AASLD guidelines, transarterial chemoembolization (TACE) is considered as the first-line choice of noncurative therapy for HCC (Bruix and Sherman, 2011; 2012). Evidence from a meta-analysis of randomized controlled trials suggests that TACE should be superior to conservative treatment in HCC patients (Llovet et al., 2002; Lo et al., 2002; Llovet and Bruix, 2003). Candidates who are eligible for TACE should be at the BCLC B stage or intermediate stage (i.e., Child-Pugh class A or
B, asymptomatic, large or multifocal nodule, neither macrovascular invasion nor extrahepatic metastasis).

As for the physicians, how to select the subsequent treatment modalities after initial TACE session is a clinically important issue. However, until now, there is no relevant consensus. Should we continue to follow the BCLC staging system or to establish a new decision making algorithm? Theoretically, there are some alternative approaches, such as repeated TACE, combined with sorafenib, followed by hepatic resection or RFA, or other promising drugs or procedures. First, the investigators from the Medical Universities of Vienna developed and validated the Assessment for Retreatment with TACE (ART) score in HCC patients to predict the necessity of repeated TACE (Sieghart et al., 2013). The ART score included radiologic tumor response and impairment of liver function (Child-Pugh score and aspartate aminotransferase) after the first TACE. Based on the ART scoring system, we could identify the patients who would have a dismal prognosis if retreated with a second TACE session. Second, the evidence from several meta-analyses suggested the advantages of TACE in combination with sorafenib over TACE alone in terms

${ }^{1}$ Meta-analysis Study Interest Group, ${ }^{2}$ Department of Stomatology, ${ }^{3}$ Department of Gastroenterology, General Hospital of Shenyang Military Area, Shenyang, China \& Equal contributors *For correspondence: guo_xiao_zhong@126.com,tangylong2009@foxmail. com,xingshunqi@126.com 
of overall survival (OS), time to progression, objective response rate, and progression free survival (Fu et al., 2014; Liu et al., 2014; Zhang et al., 2014). Thus, a combination of sorafenib should be attempted in patients at a relatively late stage of HCC. On the other hand, there is a possibility of HCC downstaging after initial TACE session, in which curative therapy became indicative and the patients would obtain more survival benefits. Indeed, the evidence from several meta-analyses suggested the superiority of TACE in combination with RFA over TACE alone (Cao et al., 2014). Considering that the OS would be better in patients undergoing hepatic resection than in those undergoing RFA (Qi et al., 2014), further studies should be necessary to explore the effectiveness of hepatic resection after initial TACE. Herein, we conducted a meta-analysis to explore this issue.

\section{Materials and Methods}

This work was registered with PROSPERO (registration number: CRD42015026157).

\section{Search strategy}

The search strategy had been described in three previous meta-analyses by our team (Qi et al., 2015a; Qi et al., 2015b; Wang et al., 2015). The first meta-analysis compared the OS of TACE versus hepatic resection for the initial treatment of HCC (Qi et al., 2015b); the second one compared the postrecurrence survival of TACE versus hepatic re-resection for the treatment of recurrent HCC after initial resection (Wang et al., 2015); and the third one compared the outcomes of hepatic resection alone versus in combination with pre- and post-operative TACE for the treatment of HCC (Qi et al., 2015a). By comparison, the present study compared the OS of TACE alone versus hepatic resection after initial TACE. Although the study objectives were different among studies, the study population was almost similar. Given that our search items were very extensive, they should be also applicable to the present study. Briefly, we searched the PubMed, EMBASE, and Cochrane Library databases by using the following search items: ("hepatectomy" OR "liver resection" OR "hepatic resection" OR "liver surgery" OR "hepatic surgery") AND ("TACE" OR "transarterial chemoembolization") AND ("HCC" OR "hepatocellular carcinoma" OR "hepatic carcinoma"). The last search was performed on December 18, 2014.

\section{Study selection}

If the studies compared the OS between HCC patients undergoing hepatic resection after initial TACE versus those undergoing TACE alone, they would be considered. The exclusion criteria should be as follows: 1) duplicate papers among databases or redundant publications; 2) narrative or systematic reviews or study protocols; 3) comments; 4) experimental studies; 5) case reports; 6) hepatic metastases; 7) mixed malignancies; 8) non-comparative studies; and 9) no comparison between TACE plus hepatic resection versus TACE alone.

Type of study design was not restricted. Either randomized or nonrandomized studies were eligible in the systematic review. Publication status and language were not restricted. If two or more papers by the same study team had the overlapping data, only one paper with more adequate data and/or a longer enrollment period would be included.

\section{Data extraction}

The following data were extracted: the first author, publication year, country, enrollment period, study design, study population, number of patients, sex, Child-Pugh class, and tumor size of HCC cases, TACE methods, selection criteria for hepatic resection, and OS rate. If only Kaplan-Meier curves were presented, we extracted the cumulative 1-,3-, and 5-year survival rates by using the Distance Tool in the Measurements menu of Foxit PDF Reader software (Foxit Cooperation, California, USA). This software was freely downloaded.

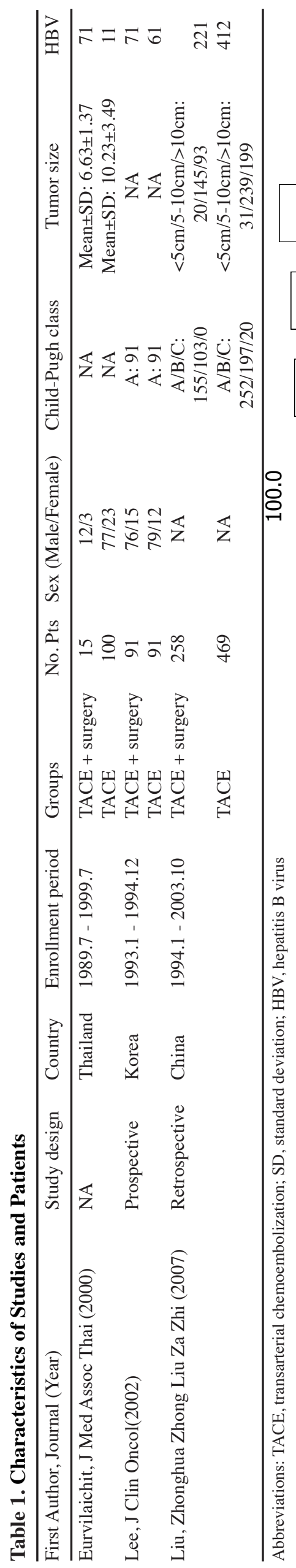




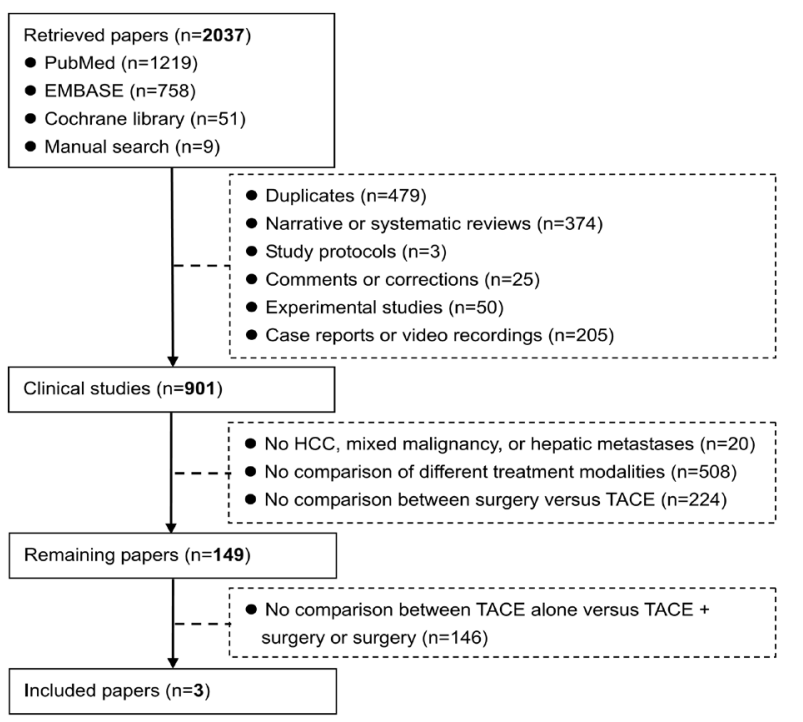

Figure 1. Flowchart of Study Inclusion

Study quality

The study quality was evaluated according to the Newcastle-Ottawa scale for cohort studies (Wells et al.), as follows:

1) Selection section: representativeness of hepatic resection after initial TACE.

2) Selection section: selection of TACE alone.

3) Selection section: ascertainment of hepatic resection after initial TACE.

4) Selection section: demonstration that outcome of interest was not present at start of study.

5) Comparability section: comparability of cohorts on the basis of the design or analysis.

6) Outcome section: assessment of outcome.

7) Outcome section: was follow-up long enough for outcomes to occur.

8) Outcome section: adequacy of follow up of cohorts.

\section{Data analysis}

Meta-analyses were performed by the statistical package Review Manager version 5.3.5 (Copenhagen, The Nordic Cochrane Center, The Cochrane Collaboration, 2014). Only random-effects models were employed. Hazard ratios (HRs) with 95\% confidence intervals (95\%CIs) were pooled by using the calculation sheets developed by Tierney et al.(Tierney et al., 2007). Heterogeneity was assessed by using the $\mathrm{I}^{2}$ statistic and the Chi-square test. $\mathrm{I}^{2}>50 \%$ or $P<0.10$ was considered to represent a significant heterogeneity. Publication bias was not evaluated due to a small number of included studies. If all studies laid within $95 \% \mathrm{CI}$, there was no proof of publication bias. Otherwise, there was a proof of publication bias.

\section{Results}

Overall, 2037 papers were initially retrieved, of which 3 were included (Eurvilaichit and Kanjanapitak, 2000; Lee et al., 2002; Liu et al., 2007) (Figure 1). Study characteristics were shown in Table 1. All of them were cohort studies. All of them were conducted in Asian countries, including Thailand $(\mathrm{n}=1)$, Korea $(\mathrm{n}=1)$, and China $(n=1)$. Methods for TACE procedures were shown in Supplementary Table 1. Selection criteria for hepatic resection were shown in Supplementary Table 2. Study quality assessment was shown in Supplementary Table 3. The quality of all included studies was not good.

The meta-analysis demonstrated a significantly better OS in patients undergoing hepatic resection after initial TACE than in those undergoing TACE alone $(\mathrm{HR}=0.63,95 \% \mathrm{CI}=0.52-0.76, P<0.00001)$ (Figure 2). The heterogeneity among studies was not statistically significant $\left(P=0.96 ; \mathrm{I}^{2}=0 \%\right)$.

\section{Discussion}

Although TACE is a mainstay treatment option for HCC, the selection of treatment modalities after initial TACE session remains unclear. Our meta-analysis found that the OS was significantly higher in patients undergoing hepatic resection after initial TACE than in those undergoing TACE alone, which favored the sequential use of hepatic resection after initial TACE for the treatment of HCC. Indeed, all of the included studies suggested a trend towards the survival benefit of hepatic resection after initial TACE. There was no statistically significant heterogeneity among studies. Therefore, we believed that our statistical results should be stable. However, we should acknowledge the following limitations. First, all of the included studies were not randomized controlled studies. There was a potential bias in the treatment selection. Further randomized studies should be warranted to confirm this finding. Second, there were only a small number of included studies. Subgroup analyses were hardly performed to identify the accurate candidates for hepatic resection according to the prognostic factors of HCC. Third, all of the included studies were from Asia. Given the geographical difference of HCC (Wallace et al., 2015), these findings should be also validated in Western countries.

In an individual study by Lee et al., the survival benefit

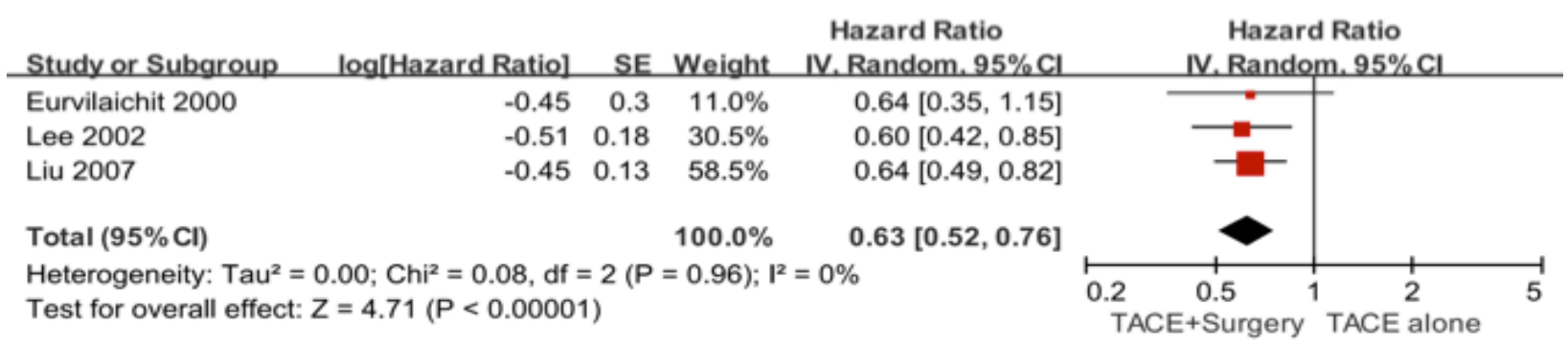

Figure 2. Forest Plot Comparing the Overall Survival between Hepatic Resection after Initial TACE Versus TACE Alone Groups 
Yu-Long Tang et al

of hepatic resection after initial TACE should be attributed to the subgroup of patients at less advanced tumor stages (Lee et al., 2002). The investigators conducted the subgroup analyses according to the International Union Against Cancer (UICC) stage and the Cancer of the Liver Italian Program (CLIP) scoring system. There was a significant survival benefit of hepatic resection after initial TACE in HCC patients with UICC T1-2 $(P=0.0058)$, rather than in those with UICC T3 $(P=0.7512)$. Similarly, there was a significant survival benefit of hepatic resection after initial TACE in HCC patients with CLIP score 0 $(P=0.0027)$, rather than in those with CLIP score 1-2 $(P=0.5366)$. Taken together, the subgroup analyses were very important to design the randomized controlled trial in future. The possible participants should be restricted to the patients at early stage of HCC. However, another two included papers by Eurvilaichit and Liu did not conduct any subgroup analyses (Eurvilaichit and Kanjanapitak, 2000; Liu et al., 2007). Therefore, future studies should confirm the findings in such patients.

Our previous meta-analysis study had compared the outcomes of pre-operative TACE plus hepatic resection versus hepatic resection alone for HCC (Qi et al., 2015a). Adjunctive use of per-operative TACE did not improve the outcome of HCC patients initially treated with hepatic resection. Notably, in this setting, the participants who underwent hepatic resection as an initial treatment modality should be at a relatively early stage of HCC. By comparison, the present study selected a different control group (i.e., TACE alone). Also, the present study had a different study population (i.e., patients who underwent TACE as an initial treatment modality should be at a relatively late stage of $\mathrm{HCC}$ ). Accordingly, their findings should be appropriate for different study population.

In conclusion, the adjunctive use of hepatic resection could improve the OS of HCC patients treated with initial TACE. The appropriateness of hepatic resection after initial TACE session should be fully evaluated in a timely fashion. However, given the limitation of our meta-analysis and quality of included studies, further randomized controlled trials should confirm these findings.

\section{Acknowledgements}

This study is partially granted by the Doctoral Program of Liaoning Province (No. 20141176).

\section{References}

Bruix J, Sherman M (2011). Management of hepatocellular carcinoma: an update. Hepatology, 53, 1020-2.

Cao JH, Zhou J, Zhang XL, et al (2014). Meta-analysis on radiofrequency ablation in combination with transarterial chemoembolization for the treatment of hepatocellular carcinoma. J Huazhong Univ Sci Technolog Med Sci, 34, 692-700.

EASL-EORTC (2012). clinical practice guidelines: management of hepatocellular carcinoma. J Hepatol, 56, 908-43.

El-Serag HB (2011). Hepatocellular carcinoma. $N$ Engl J Med, 365, 1118-27.

Eurvilaichit C, Kanjanapitak A (2000). Hepatocellular carcinoma : treated with hepatic arterial embolization, an analysis of prognostic features in 150 cases. J Med Assoc Thai, 83, 983-91.

Forner A, Llovet JM, Bruix J (2012). Hepatocellular carcinoma. Lancet, 379, 1245-55.

Fu QH, Zhang Q, Bai XL, et al (2014). Sorafenib enhances effects of transarterial chemoembolization for hepatocellular carcinoma: a systematic review and meta-analysis. J Cancer Res Clin Oncol, 140, 1429-40.

Lee HS, Kim KM, Yoon JH, et al (2002). Therapeutic efficacy of transcatheter arterial chemoembolization as compared with hepatic resection in hepatocellular carcinoma patients with compensated liver function in a hepatitis B virus-endemic area: a prospective cohort study. J Clin Oncol, 20, 4459-65.

Liu L, Chen H, Wang M, et al (2014). Combination therapy of sorafenib and TACE for unresectable HCC: a systematic review and meta-analysis. PLoS One, 9, 91124.

Liu YM, Qin H, Wang CB, et al (2007). Comparision of different interventional therapies for primary liver cancer. Zhonghua zhong liu za zhi [Chinese journal of oncology], 29, 232-5.

Llovet JM, Bruix J (2003). Systematic review of randomized trials for unresectable hepatocellular carcinoma: Chemoembolization improves survival. Hepatology, 37, 429-42.

Llovet JM, Real MI, Montana X, et al (2002). Arterial embolisation or chemoembolisation versus symptomatic treatment in patients with unresectable hepatocellular carcinoma: a randomised controlled trial. Lancet, 359, 1734-9.

Lo CM, Ngan H, Tso WK, et al (2002). Randomized controlled trial of transarterial lipiodol chemoembolization for unresectable hepatocellular carcinoma. Hepatology, 35, 1164-71.

Qi X, Liu L, Wang D, et al (2015a). Hepatic resection alone versus in combination with pre- and post-operative transarterial chemoembolization for the treatment of hepatocellular carcinoma: A systematic review and metaanalysis. Oncotarget, In press.

Qi X, Tang Y, An D, et al (2014). Radiofrequency ablation versus hepatic resection for small hepatocellular carcinoma: a meta-analysis of randomized controlled trials. J Clin Gastroenterol, 48, 450-7.

Qi X, Wang D, Su C, et al (2015b). Hepatic resection versus transarterial chemoembolization for the initial treatment of hepatocellular carcinoma: A systematic review and metaanalysis. Oncotarget, 6, 18715-33.

Sieghart W, Hucke F, Pinter M, et al (2013). The ART of decision making: retreatment with transarterial chemoembolization in patients with hepatocellular carcinoma. Hepatology, 57, 2261-73.

Tierney JF, Stewart LA, Ghersi D, et al (2007). Practical methods for incorporating summary time-to-event data into metaanalysis. Trials, $\mathbf{8}, 16$.

Wallace MC, Preen D, Jeffrey GP, et al (2015). The evolving epidemiology of hepatocellular carcinoma: a global perspective. Expert Rev Gastroenterol Hepatol, 9, 765-79.

Wang DY, Liu L, Qi XS, et al (2015). Hepatic re-resection versus transarterial chemoembolization for the treatment of recurrent hepatocellular carcinoma after initial resection: a systematic review and meta-analysis. Asian Pac J Cancer Prev, 16, 5573-8.

Wells GA, Shea B, O'Connell D, et al The Newcastle-Ottawa Scale (NOS) for assessing the quality of nonrandomised studies in meta-analyses. http://www.ohri.ca/programs/ clinical_epidemiology/oxford.asp.

Zhang L, Hu $P$, Chen X, et al (2014). Transarterial chemoembolization (TACE) plus sorafenib versus TACE for intermediate or advanced stage hepatocellular carcinoma: a meta-analysis. PLoS One, 9, 100305. 